\title{
Guest Editorial Foreword to the Special Issue on Data Fusion
}

\section{INTRODUCTION}

D ATA fusion emerged as a new topic in the late 1980s, but it was only by the first half of the following decade that the availability of remotely sensed data in digital form by different sources allowed the consideration of remote-sensing data fusion. At that point, the Data Fusion Technical Committee (DFTC) of the IEEE Geoscience and Remote Sensing Society recognized the need for a Special Issue on the IEEE Transactions on Geoscience and Remote Sensing about "data fusion," which was published in May 1999. That pioneering issue brought to the attention of many researchers the need for an increased effort toward the joint exploitation of multiple data or information sources.

In the following years, and until now, some of the fields highlighted in that issue have come to full maturity. This is the case for multitemporal data fusion, which is currently investigated by a very active community gathered by Bruzzone and Smits [1] in the MULTITEMP Workshops.

Many other research fields have however emerged, as results of the continuous improvement in data quality and quantity, and the fast changing electronic and optical technologies, that allow recording, transmitting, and storing of a huge amount of information. For instance, spatial and spectral resolutions of remotely sensed data are steadily increasing, going toward very high-resolution sensors, both in the spatial and in the spectral sense. This requires combined spectral and spatial analyses, and fusion of features extracted and selected at different scales from the same data set, as in [2].

Moreover, the third dimension is no longer an option for remotely sensed data, and multiple bidimensional view analysis of the same area and multiple 3-D data comparison and combination are some new faces of the same data-fusion problem [3], [4].

At a higher level, feature fusion has also been increased by the larger CPU power and memory capacity of modern processors, which is still to be pushed by the use of multiple CPUs and grid/distributed computing [5].

Fusion of geographical information and remotely sensed data is requiring fusion architectures that are fully aware of the multiple levels of "fusion" discussed in [6], and feature extraction and combination at a geometrical level is felt as a possible common practice in the near future to improve classification [7], change detection [8], and also preanalysis

Color versions of one or more of the figures in this paper are available online at http://ieeexplore.ieee.org.

Digital Object Identifier 10.1109/TGRS.2008.919761 processing steps [9]. It is not certainly by chance that ENVI has recently announced the release of a feature extraction toolbox.

Similarly, the contemporary use of 2-D features and 3-D data was suggested in [10] using geographic information system data, but this is just the simplest part of the work. As a matter of fact, 2-D features and 3-D data may concur to improve the information extracted from each other, as considered for instance in [11]. Three-dimensional building shapes help in distinguishing patches of the same material covering objects at different heights or in correcting errors in patches with different reflectances but at the same level [12].

For a recent general survey paper with classification on information fusion, please refer to [13].

\section{DATA Fusion AND REMOTELY SENSED DATA PROCESSING}

In an effort to highlight these and other newly emerging research lines, this Special Issue on the topic of "data fusion" provides to interested readers a sort of quick look on approaches currently evolving. The effort of the Guest Editors, and the purpose of this Special Issue, is to put the papers that form the core of the issue in a more general framework, thus helping those who are interested to the topic to easily pick up their choice. To this aim, the remaining part of this Special Issue is organized according to the schematic processing flow in Fig. 1, starting from data and leading to information through a number of mandatory and optional steps.

\section{A. Registration}

As shown in Fig. 1, nowadays, the most realistic assumption for a problem facing the use of remotely sensed data is that more data sources are available. They might come from more platforms, more sensors onboard the same platform, ancillary data sources, and so on. This situation usually requires the user to manage a multisensor and, possibly, a multitemporal data set. The first problem to be faced is coregistration. While this processing step has been analyzed for a long time in technical literature, it remains a crucial step in numerous applications and still gathers a lot of attention [14]. As a matter of fact, every kind of data requires some specific development: SAR data [15], [16] or multispectral (MS) data [17] and hyperspectral data [18]. For an increased accuracy, subpixel registration might be the ultimate step in the field [19], [20].

In this Special Issue, the paper proposed by Cariou and Chehdi [21] tries to solve one of the problems that are still open: the lack of adequate ancillary data. In particular, the use of "mutual information" between the data and a reference 


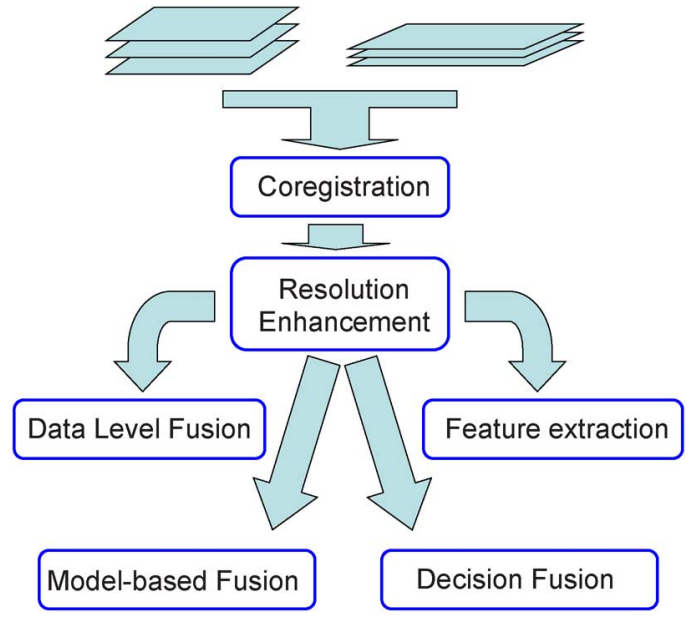

Fig. 1. Schematic processing flow for remotely sensed data processing.

orthophoto is shown as a suitable mean to obtain accurate georeferencing of pushbroom scanners' data.

\section{B. Resolution Enhancement}

The spatial resolution is one key characteristic of remotesensing data. Be it high or low, uniform or not, it directly impacts the results of the processing and the potential applications. Consequently, a lot of energy is devoted to tackle this limitation. By merging different sources of information, data fusion can actually enhance the available resolutions.

1) Pansharpening: The synthesis of MS images at a higher spatial resolution can be achieved by exploiting an alternate high-resolution image acquired in another modality. These synthetic images should be similar to the MS images that would have been observed with a sensor at the higher resolution [22], [23]. When the higher resolution image is panchromatic (Pan), i.e., a single wide spectral-band image acquired across the visible and possibly near-infrared wavelengths, this fusion process is usually called pansharpening of MS images.

Spaceborne sensors, such as SPOT, Ikonos, or QuickBird, provide images with different characteristics: on the one hand, images with high spectral resolution but low spatial resolution, and on the other hand, images with low spectral resolution but high spatial resolution. Several works have demonstrated the usefulness of fused products offering high spectral and spatial resolutions at the same time in various environmental applications [24], [25]. Pansharpened products are becoming very popular (for example, Google Earth), and data providers are offering higher and higher amounts of them at lower and lower costs. The Data Fusion Contest organized by the DFTC at the IEEE Geoscience and Remote Sensing Symposium 2006 [26] was meant as the ultimate word on the subject, after the book by Wald [27] and the authoritative papers by Alparone et al. [28], [29].

As recalled in [26], a variety of pansharpening techniques take advantage of the complementary characteristics of spatial and spectral resolutions of the data [30], [31]. Among them, component substitution (CS) methods [32] are attractive because they are fast and easy to implement. When exactly three MS bands are concerned, the most widely used CS fusion method is based on the intensity-hue-saturation (IHS) transformation [33]. The spectral bands are resampled and coregistered to the Pan image before the IHS transformation is applied. The smooth intensity component $I$ is substituted with the highresolution Pan and transformed back to the spectral domain via the inverse IHS transformation.

Multiresolution analysis (MRA) is the alternative; it provides effective tools like wavelets and Laplacian pyramids to help carry out data-fusion tasks. MRA-based fusion requires the definition of a model establishing how the missing high-pass information is to be extracted from the Pan image and then injected into the MS bands [23], [34]-[36].

In this Special Issue, the necessity to take physical considerations into account when designing a pansharpening algorithm is clearly established by Thomas et al. [37]. A pansharpening method using a multiscale mapped least squares support vector machine (SVM) is proposed by Zheng et al. [38], whereas the popular MRA based on wavelet analysis is brought one step forward by Shah et al. [39] by using contourlet decomposition, for a better representation of edges. Finally, Aanaes et al. [40] introduced a model-based fusion and describe a novel approach to improve the spatial resolution even when no simple ratio between the coarse and the finer resolution data is available.

2) Subpixel Processing: Beyond the continuous improvement of the sensors in terms of spatial resolution, there is a need for even more accurate processing and analysis; subpixel processing is on stake. As already mentioned, this addresses the task of registration. Two more papers of this Special Issue can fit in this category.

1) In the case of hyperspectral images, Gu et al. [41] propose using some spectral unmixing and superresolution mapping to enhance the spatial resolution while preserving the spectral diversity.

2) Beyond spectral unmixing, subpixel classification is addressed by Robin et al. [42]. This ill-posed problem is solved by injecting some further information (time series) and some prior structural knowledge.

3) Downscaling: It is worth noting that spatial enhancement sometimes is based on fusion of ancillary information, even if the ancillary information has sparse and somehow spatially irregular sampling on the ground. This is the case for many models for hydrology or environmental analysis, whose inputs are both remotely sensed data, usually at coarse spatial resolution, and locally very detailed in situ measurements. It is increasingly important to develop methodologies aimed at combining these two very different sources of information and to provide jointly spatially enhanced and calibrated data by means of a downscaling approach. This is what is proposed by Kaheil et al. [43] for soil moisture measurements.

\section{Data Level Fusion}

The first way to exploit the georeferenced and spatially enhanced data sets achieved by means of the previous processing step is to directly proceed to information extraction. Joint per-pixel analysis is usually labeled as "data level fusion." Typical outputs are land-cover/land-use classification in the 
case of multisensor Earth observation data and change detection maps for multitemporal data sets. A good example of these techniques is presented in [44]. In this Special Issue, an image fusion technique based on partial differential equation is proposed. By means of diffusion and inverse diffusion, the information from multiple stocks of seismic measurements is enhanced with respect to the extraction of fault structures.

A similar approach for the fusion of multiple runs from the same (kind of) sensor is proposed in [45], where multiple inverse synthetic aperture radar measurements are combined to a more effective target detection. It is a very interesting approach that exploits the spatial frequency space where SAR images are formed as the framework in which data fusion is implemented. Proposed rules allow the combination of sensors with different spatial and frequency resolutions by means of the proposed matrix Fourier transform algorithm.

A relatively less new, but still active, research line is instead the one referring to the exploitation of multisensor data. This Special Issue brings in an excellent example. In [46], the combination of different properties of the atmosphere, as they are measured by multiple sensors, is used to achieve better characterization of the aerosol properties than that available from any single sensor. The fusion rules are based on a priori knowledge on the meteorological phenomena and their effect at different wavelengths and on different measured quantities.

On the same subject, and on one of the innovative research lines in data fusion highlighted in the introduction, Dalponte et al. [47] show the combination of 2- and 3-D data sets. In particular, in this Special Issue, hyperspectral and lidar data are jointly considered for forest analysis. The approach is very similar to a stack of the 2 - and 3 -D data, but a feature extraction is run before in order to select the best information for the task.

Finally, this Special Issue carries an example of data fusion for change detection. The paper by Mercier et al. [48] is extracting change areas by comparison of statistical behavior of preand postdata sets that might be very different as for acquisition conditions. The paper works in the opposite direction than most of the similar works. It tries to define the similarity in unchanged areas and, in the statistical sense, to learn when a change has happened as a nonstationary statistics is recognized.

\section{Feature Extraction}

Data fusion at the feature level consists in extracting different, and most of the time complementary, features from the data; these features become the inputs of one single processing (e.g., a classifier). These features can be of different natures (statistical, geometrical, and so on); they can be extracted from the initial data or after some transform.

In this Special Issue, Roy et al. [49] present an application of how such features can be extracted and merged for the analysis of microbarographs. In this case, a transformed representation of the data is used, namely, the Huang-Hilbert transform, decomposing nonstationary signals in order to highlight moving trends and break it into locally orthogonal components.

In remote-sensing imagery, an important trend consists in using simultaneously the spectral information (multi- or hy- perspectral data) and the spatial information [50]-[53]. This is particularly helpful for the classification of man-made structures where the shape of the objects actually helps recognize the corresponding structures, such as in urban areas.

\section{E. Decision Fusion}

For most of the usual tasks in remote sensing (detection, classification, segmentation, etc.), an abundant literature can be found, with numerous algorithms being proposed. Most of the time, every algorithm has its own merits, and it is rare to find one that systematically outperforms all the others. As a consequence, a key issue in data fusion consists in simultaneously using different algorithms, trying to take advantage of their respective merits, thus increasing the overall performances. This process is generally referred to as "decision fusion" [54]-[56]. Multiple classifier systems (MCSs) can also fit in this framework (see [57] for a recent overview of MCS in remote sensing).

The fusion can look for complementarity between the algorithms when they are based on different properties (for instance, morphological and statistical detectors [58]). On the contrary, it can look for redundancy to decrease the false-alarm rate [59], assuming that the noise is not redundant. When the different sources of information agree, the final output remains the same as with an individual algorithm, but the confidence in this result increases. When they disagree, there is a chance to actually increase the individual performances by picking the right output up. The key point hence lies in defining the reliability of every output in order to select the most reliable one [60]. This can be an adaptive criterion or a criterion based on some prior knowledge.

In this Special Issue, the task of target recognition in the frame of hyperspectral imagery is addressed by Prasad and Bruce [61]. The initial hyperspectral space is partitioned into contiguous subspaces based on the optimization of a performance metric. Local classification decisions are then met in every subspace using an MCS. Decision fusion with an adaptive weight assignment (based on the strengths of individual local classifiers) makes the final decision.

Waske and van der Linden [62] propose a joint classification of multiple segmentation levels from multisensor imagery using SAR and optical data which are first separately segmented, creating independent aggregation levels at different scales. Each individual level from the two sensors is preclassified by an SVM. The original outputs of each SVM, i.e., images showing the distances of the pixels to the hyperplane fitted by the SVM, are used in a decision fusion to determine the final classes.

On a different level of decision fusion, in [63], the stress is on classifier outputs' fusion by means of robust algorithms. The author introduces two different algorithms for fault-tolerant classifier combination. The goal is to find an approach that is sufficiently robust to work well in case of corrupted classification maps in input. The overall methodology is tested in a multisensor environment, exploiting optical, passive, and active radar data acquired onboard the ENVISAT satellite. 


\section{F. Fuzzy-Model-Based Fusion}

Fusion operators based on fuzzy models and/or fuzzy combination rules play a major role. As a matter of fact, these operators can handle imprecise or uncertain information, which is very important when different sources of information are conflictual. They also allow one to postpone the final decision to every last step of the processing, thus preserving as much information as possible, as long as possible. Another advantage is the possibility offered to handle semantic data and to model some very rough expert's knowledge. Finally, by using fuzzy distributions, the models are very flexible and not too sensitive to parameter settings.

These models have already proved their efficiency in various remote-sensing applications [59], [64], [65]. For a milestone paper by Bloch, please refer to [66].

In this Special Issue, following a previous work, Milisavljevic and Bloch [67] present different fusion strategies based on possibilistic models or belief functions for antipersonnel mine detection [68]. Fuzzy fusion rules offer a wide range of behaviors (indulgent, severe, cautious, etc.), providing different properties in order to match the user's expectations. Kallel et al. [69] address the analysis of vegetation index and propose a cautious-adaptive combination rule based on belief functions.

\section{CONCLUSION}

New research fields are emerging, and new forces are working toward new data-fusion algorithms, architectures, and solutions. This Special Issue was meant to give them an option to discuss their achievements and set a further mileage stone in the DFTC's history.

The overwhelming response to the call for papers (more than 45 submitted papers) showed that we were right. The strong selection procedure provided us with an excellent table of content. It sets the scene for the next years, but we already forecast a new issue in the near future. For the moment, enjoy your reading!

\section{ACKNOWLEDGMENT}

The Guest Editors would like to thank the IEEE Geoscience and Remote Sensing Society AdCom for its support, all the members of the Data Fusion Technical Committee for their contribution to keep our community so active, and the numerous reviewers who provided extremely valuable help in putting this very exciting issue together.

\section{PaOlo Gamba, Guest Editor \\ University of Pavia \\ Department of Electronics \\ 27100 Pavia, Italy}

Jocelyn Chanussot, Guest Editor

Grenoble Institute of Technology

Grenoble Images Speech Signals and Automatics Laboratory 38000 Grenoble, France

\section{REFERENCES}

[1] L. Bruzzone and P. Smits, "Analysis of multi-temporal remote sensing images," in Proc. MULTITEMP. Singapore: World Scientific, 2001.

[2] A. Plaza, P. Martinez, J. Plaza, and R. Perez, "Dimensionality reduction and classification of hyperspectral image data using sequences of extended morphological transformations," IEEE Trans. Geosci. Remote Sens., vol. 43, no. 3, pp. 466-479, Mar. 2005.

[3] P. Gamba, F. Dell'Acqua, and B. Houshmand, "Comparison and fusion of LIDAR and INSAR digital elevation models over urban areas," Int. J. Remote Sens., vol. 24, no. 22, pp. 4289-4300, Nov. 2003.

[4] R. Bolter, "Buildings from SAR: Detection and reconstruction of buildings from multiple view high resolution interferometric SAR data," Ph.D. dissertation, Tech. Univ. Graz, Graz, Austria, 2001.

[5] J. P. A. Plaza, D. Valencia, and C.-I Chang, "Parallel implementation of endmember extraction algorithms from hyperspectral data," IEEE Geosci. Remote Sens. Lett., vol. 3, no. 3, pp. 334-338, Jul. 2006.

[6] C. Pohl and J. V. Genderen, "Multisensor image fusion in remote sensing: Concepts, methods and applications," Int. J. Remote Sens., vol. 19, no. 5, pp. 823-854, Mar. 1998.

[7] J. Chanussot, J. Benediktsson, and M. Fauvel, "Classification of remote sensing images from urban areas using a fuzzy possibilistic model," IEEE Geosci. Remote Sens. Lett., vol. 3, no. 1, pp. 40-44, Jan. 2006.

[8] P. Gamba, F. Dell'Acqua, and G. Lisini, "Change detection of multitemporal SAR data in urban areas combining feature-based and pixelbased techniques," IEEE Trans. Geosci. Remote Sens., vol. 44, no. 10, pp. 2820-2827, Oct. 2006

[9] S. Growe and R. Tonjes, "A knowledge based approach to automatic image registration," in Proc. IEEE Int. Conf. Image Process., 1997, vol. 3, pp. 23-29.

[10] N. Haala, "Combining multiple data sources for urban data acquisition," in Proc. Photogrammetric Week, Stuttgart, Germany, 1999, pp. 329-339.

[11] F. Tupin and M. Roux, "Markov random fields for DTM extraction," in Proc. IEEE/ISPRS Joint Workshop Remote Sens. Data Fusion Over Urban Areas, Rome, Italy, 2001, pp. 95-99.

[12] V. Madhok and D. Landgrebe, "Supplementing hyperspectral data with digital elevation," in Proc. IEEE Geosci. Remote Sens. Symp., 1999, vol. I, pp. 59-61.

[13] L. Valet, G. Mauris, and P. Bolon, "A statistical overview of recent literature in information fusion," IEEE Aerosp. Electron. Syst. Mag., vol. 16, no. 3, pp. 7-14, Mar. 2001.

[14] A. Wong and D. Clausi, "ARRSI: Automatic registration of remotesensing images," IEEE Trans. Geosci. Remote Sens., vol. 45, no. 5, pp. 1483-1493, May 2007.

[15] E. Sansosti, P. Berardino, M. Manunta, F. Serafino, and G. Fornaro, "Geometrical SAR image registration," IEEE Trans. Geosci. Remote Sens., vol. 44, no. 10, pp. 2861-2870, Oct. 2006.

[16] F. Serafino, "SAR image coregistration based on isolated point scatterers," IEEE Geosci. Remote Sens. Lett., vol. 3, no. 3, pp. 354-358, Jul. 2006.

[17] J. Kern and M. Pattichis, "Robust multispectral image registration using mutual-information models," IEEE Trans. Geosci. Remote Sens., vol. 45, no. 5, pp. 1494-1505, May 2007.

[18] H. Erives and G. Fitzgerald, "Automatic subpixel registration for a tunable hyperspectral imaging system," IEEE Geosci. Remote Sens. Lett., vol. 3, no. 3, pp. 397-400, Jul. 2006.

[19] J. Inglada, V. Muron, D. Pichard, and T. Feuvrier, "Analysis of artifacts in subpixel remote sensing image registration," IEEE Trans. Geosci. Remote Sens., vol. 45, no. 1, pp. 254-264, Jan. 2007.

[20] S. Leprince, S. Barbot, F. Ayoub, and J.-P. Avouac, "Automatic and precise orthorectification, coregistration, and subpixel correlation of satellite images, application to ground deformation measurements," IEEE Trans. Geosci. Remote Sens., vol. 45, no. 6, pp. 1529-1558, Jun. 2007.

[21] C. Cariou and K. Chehdi, "Automatic georeferencing of airborne pushbroom scanner images with missing ancillary data using mutual information," IEEE Trans. Geosci. Remote Sens., vol. 46, no. 5, pp. 1290-1300, May 2008.

[22] T. Ranchin and L. Wald, "Fusion of high spatial and spectral resolution images: The ARSIS concept and its implementation," Photogramm. Eng. Remote Sens., vol. 66, no. 1, pp. 49-61, Jan. 2000.

[23] T. Ranchin, B. Aiazzi, L. Alparone, S. Baronti, and L. Wald, "Image fusion-The ARSIS concept and some successful implementation schemes," ISPRS J. Photogramm. Remote Sens., vol. 58, no. 1/2, pp. 4-18, Jun. 2003.

[24] L. Wald and J.-M. Baleynaud, "Observing air quality over the city of Nantes by means of Landsat thermal infrared data," Int. J. Remote Sens., vol. 20, no. 5, pp. 947-959, Mar. 1999. 
[25] P. Sirguey, R. Mathieu, Y. Arnaud, M. Khan, and J. Chanussot, "Improving MODIS spatial resolution for snow mapping using wavelet fusion and ARSIS concept," IEEE Geosci. Remote Sens. Lett., vol. 5, no. 1, pp. 78-82, Jan. 2008.

[26] L. Alparone, L. Wald, J. Chanussot, C. Thomas, P. Gamba, and L. Mann Bruce, "Comparison of pansharpening algorithms: Outcome of the 2006 GRS-S data fusion contest," IEEE Trans. Geosci. Remote Sens., vol. 45, no. 10, pp. 3012-3021, Oct. 2006

[27] L. Wald, Data Fusion: Definitions and Architectures-Fusion of Images of Different Spatial Resolutions. Paris, France: ENSMP, 2002.

[28] L. Alparone, S. Baronti, A. Garzelli, and F. Nencini, "A global quality measurement of pan-sharpened multispectral imagery," IEEE Geosci. Remote Sens. Lett., vol. 1, no. 4, pp. 313-317, Oct. 2004.

[29] B. Aiazzi, L. Alparone, S. Baronti, and A. Garzelli, "Context-driven fusion of high spatial and spectral resolution images based on oversampled multiresolution analysis," IEEE Trans. Geosci. Remote Sens., vol. 40, no. 10 , pp. $2300-2312$, Oct. 2002.

[30] Z. Wang, D. Ziou, C. Armenakis, D. Li, and Q. Li, "A comparative analysis of image fusion methods," IEEE Trans. Geosci. Remote Sens., vol. 43, no. 6, pp. 1391-1402, Jun. 2005.

[31] M. Khan, J. Chanussot, L. Condat, and A. Montanvert, "Indusion: Fusion of multispectral and panchromatic images using the induction scaling technique," IEEE Geosci. Remote Sens. Lett., vol. 5, no. 1, pp. 98-102, Jan. 2008.

[32] B. Aiazzi, S. Baronti, and M. Selva, "Improving component substitution pansharpening through multivariate regression of MS + pan data," IEEE Trans. Geosci. Remote Sens., vol. 45, no. 10, pp. 3230-3239, Oct. 2007.

[33] T.-M. Tu, P. S. Huang, C.-L. Hung, and C.-P. Chang, "A fast intensity-hue-saturation fusion technique with spectral adjustment for IKONOS imagery," IEEE Geosci. Remote Sens. Lett., vol. 1, no. 4, pp. 309-312, Oct. 2004

[34] J. Núñez, X. Otazu, O. Fors, A. Prades, V. Palà, and R. Arbiol, "Multiresolution-based image fusion with additive wavelet decomposition," IEEE Trans. Geosci. Remote Sens., vol. 37, no. 3, pp. 1204-1211, May 1999.

[35] B. Aiazzi, L. Alparone, S. Baronti, A. Garzelli, and M. Selva, "MTFtailored multiscale fusion of high-resolution MS and Pan imagery," Photogramm. Eng. Remote Sens., vol. 72, no. 5, pp. 591-596, May 2006.

[36] M. Gonzáles Audícana, J. L. Saleta, R. G. Catalán, and R. García, "Fusion of multispectral and panchromatic images using improved IHS and PCA mergers based on wavelet decomposition," IEEE Trans. Geosci. Remote Sens., vol. 42, no. 6, pp. 1291-1299, Jun. 2004.

[37] C. Thomas, T. Ranchin, L. Wald, and J. Chanussot, "Synthesis of multispectral images to high spatial resolution: A critical review of fusion methods based on remote sensing physics," IEEE Trans. Geosci. Remote Sens., vol. 46, no. 5, pp. 1301-1312, May 2008.

[38] S. Zheng, W.-Z. Shi, J. Liu, and J. Tian, "Remote sensing image fusion using multiscale mapped LS-SVM," IEEE Trans. Geosci. Remote Sens., vol. 46, no. 5, pp. 1313-1322, May 2008.

[39] V. P. Shah, N. H. Younan, and R. L. King, "An efficient pan-sharpening method via a combined adaptive PCA approach and contourlets," IEEE Trans. Geosci. Remote Sens., vol. 46, no. 5, pp. 1323-1335, May 2008.

[40] H. Aanæs, J. R. Sveinsson, A. A. Nielsen, T. Bøvith, and J. A. Benediktsson, "Model-based satellite image fusion," IEEE Trans. Geosci. Remote Sens., vol. 46, no. 5, pp. 1336-1346, May 2008.

[41] Y. Gu, Y. Zhang, and J. Zhang, "Integration of spatial-spectral information for resolution enhancement in hyperspectral images," IEEE Trans. Geosci. Remote Sens., vol. 46, no. 5, pp. 1347-1358, May 2008.

[42] A. Robin, S. Le Hégarat-Mascle, and L. Moisan, "Unsupervised subpixelic classification using coarse-resolution time series and structural information," IEEE Trans. Geosci. Remote Sens., vol. 46, no. 5, pp. 13591374, May 2008.

[43] Y. H. Kaheil, M. K. Gill, M. McKee, L. A. Bastidas, and E. Rosero, "Downscaling and assimilation of surface soil moisture using ground truth measurements," IEEE Trans. Geosci. Remote Sens., vol. 46, no. 5, pp. 1375-1384, May 2008.

[44] S. Pop, O. Lavialle, M. Donias, R. Terebes, M. Borda, S. Guillon, and N. Keskes, "A PDE-based approach to three-dimensional seismic data fusion," IEEE Trans. Geosci. Remote Sens., vol. 46, no. 5, pp. 1385-1393, May 2008.

[45] Z. Li, S. Papson, and R. M. Narayanan, "Data-level fusion of multilook inverse synthetic aperture radar images," IEEE Trans. Geosci. Remote Sens., vol. 46, no. 5, pp. 1394-1406, May 2008.

[46] P. Gupta, F. Patadia, and S. A. Christopher, "Multisensor data product fusion for aerosol research," IEEE Trans. Geosci. Remote Sens., vol. 46, no. 5, pp. 1407-1415, May 2008.
[47] M. Dalponte, L. Bruzzone, and D. Gianelle, "Fusion of hyperspectral and LIDAR remote sensing data for classification of complex forest areas," IEEE Trans. Geosci. Remote Sens., vol. 46, no. 5, pp. 14161427, May 2008.

[48] G. Mercier, G. Moser, and S. B. Serpico, "Conditional copulas for change detection in heterogeneous remote sensing images," IEEE Trans. Geosci. Remote Sens., vol. 46, no. 5, pp. 1428-1441, May 2008.

[49] A. Roy, C.-H. Wen, J. F. Doherty, and J. D. Matthews, "Signal feature extraction from microbarograph observations using the Hilbert-Huang transform," IEEE Trans. Geosci. Remote Sens., vol. 46, no. 5, pp. 14421447, May 2008.

[50] M. Loghmari, M. Naceur, and M. Boussema, "A spectral and spatial source separation of multispectral images," IEEE Trans. Geosci. Remote Sens., vol. 44, no. 12, pp. 3659-3673, Dec. 2006.

[51] H. Viggh and D. Staelin, "Surface reflectance estimation using prior spatial and spectral information," IEEE Trans. Geosci. Remote Sens., vol. 45 no. 9, pp. 2928-2939, Sep. 2007.

[52] V. Buntilov and T. Bretschneider, "A content separation image fusion approach: Toward conformity between spectral and spatial information," IEEE Trans. Geosci. Remote Sens., vol. 45, no. 10, pp. 3252-3263, Oct. 2007.

[53] M. Fauvel, J. Benediktsson, J. Sveinsson, and J. Chanussot, "Spectral and spatial classification of hyperspectral data using SVMS and morphological profiles," in Proc. IGARSS, 2007, pp. 4834-4837.

[54] Y. Liao, L. Nolte, and L. Collins, "Decision fusion of groundpenetrating radar and metal detector algorithms-A robust approach," IEEE Trans. Geosci. Remote Sens., vol. 45, no. 2, pp. 398-409, Feb. 2007.

[55] B. Waske and J. Benediktsson, "Fusion of support vector machines for classification of multisensor data," IEEE Trans. Geosci. Remote Sens., vol. 45 , no. 12 , pp. $3858-3866$, Dec. 2007.

[56] P. Gamba, F. D. Acqua, G. Lisini, and G. Trianni, "Improved VHR urban area mapping exploiting object boundaries," IEEE Trans. Geosci. Remote Sens., vol. 45, no. 8, pp. 2676-2682, Aug. 2007.

[57] J. Benediktsson, J. Chanussot, and M. Fauvel, "Multiple classifiers in remote sensing: From basics to recent developments," in Proc. 7th Int. Workshop Multiple Classifier Syst., 2007, pp. 501-512.

[58] G. Ginolhac, J. Chanussot, and C. Hory, "Morphological and statistical approaches to improve detection in the presence of reverberation," IEEE J. Ocean. Eng., vol. 30, no. 4, pp. 881-899, Oct. 2005.

[59] J. Chanussot, G. Mauris, and P. Lambert, "Fuzzy fusion techniques for linear features detection in multitemporal SAR images," IEEE Trans. Geosci. Remote Sens., vol. 37, no. 3, pp. 1292-1305, May 1999.

[60] M. Fauvel, J. Chanussot, and J. Benediktsson, "Decision fusion for the classification of urban remote sensing images," IEEE Trans. Geosci. Remote Sens., vol. 44, no. 10, pp. 2828-2838, Oct. 2006.

[61] S. Prasad and L. Mann Bruce, "Decision fusion with confidence-based weight assignment for hyperspectral target recognition," IEEE Trans. Geosci. Remote Sens., vol. 46, no. 5, pp. 1448-1456, May 2008

[62] B. Waske and S. van der Linden, "Classifying multilevel imagery from SAR and optical sensors by decision fusion," IEEE Trans. Geosci. Remote Sens., vol. 46, no. 5, pp. 1457-1466, May 2008.

[63] A. V. Bogdanov, "Neuroinspired architecture for robust classifier fusion of multisensor imagery," IEEE Trans. Geosci. Remote Sens., vol. 46, no. 5, pp. 1467-1487, May 2008.

[64] F. Tupin, I. Bloch, and H. Maitre, "A first step toward automatic interpretation of SAR images using evidential fusion of several structure detectors," IEEE Trans. Geosci. Remote Sens., vol. 37, no. 3, pp. 1327-1343, May 1999.

[65] S. Le Hégarat-Mascle, I. Bloch, and D. Vidal-Madjar, "Application of Dempster-Shafer evidence theory to unsupervised classification in multisource remote sensing," IEEE Trans. Geosci. Remote Sens., vol. 35, no. 4, pp. 1018-1031, Jul. 1997.

[66] I. Bloch, "Information combination operators for data fusion: A comparative review with classification," IEEE Trans. Syst., Man, Cybern. A, Syst., Humans, vol. 26, no. 1, pp. 52-67, Jan. 1996.

[67] N. Milisavljević and I. Bloch, "Sensor fusion in anti-personnel mine detection using a two-level belief function model," IEEE Trans. Syst., Man, Cybern. C, Appl. Rev., vol. 33, no. 2, pp. 269-283, May 2003.

[68] N. Milisavljević and I. Bloch, "Possibilistic versus belief function fusion for antipersonnel mine detection," IEEE Trans. Geosci. Remote Sens., vol. 46, no. 5, pp. 1488-1498, May 2008.

[69] A. Kallel, S. Le Hégarat-Mascle, L. Hubert-Moy, and C. Ottlé, "Fusion of vegetation indices using continuous belief functions and cautious-adaptive combination rule," IEEE Trans. Geosci. Remote Sens., vol. 46, no. 5, pp. 1499-1513, May 2008. 


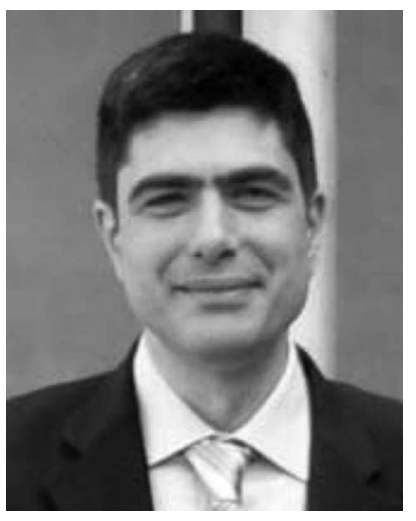

Paolo Gamba (S'91-M'93-SM'00) received the Laurea (cum laude) and Ph.D. degrees in electronic engineering from the University of Pavia, Pavia, Italy, in 1989 and 1993, respectively.

$\mathrm{He}$ is currently an Associate Professor of telecommunications with the Department of Electronics, University of Pavia. He has published more than 40 papers in peer-review journals on urban remote sensing and presented more than 100 papers at workshops and conferences.

Dr. Gamba has been the Guest Editor of special issues of the ISPRS Journal of Photogrammetry and Remote Sensing, IEEE TRansactions on Geoscience and Remote Sensing, International Journal of Information Fusion, and Pattern Recognition Letters. He serves as an Associate Editor for the IEEE Geoscience And Remote Sensing Letters. He is the Organizer and the Technical Chair of the biennial Geoscience and Remote Sensing Society (GRSS)/International Society for Photogrammetry and Remote Sensing Joint Workshops on "Remote Sensing and Data Fusion Over Urban Areas" from 2001 to 2007. He has been the Chair of Technical Committee 7 "Pattern Recognition in Remote Sensing" of the International Association for Pattern Recognition from October 2002 to October 2004. He is currently the Chair of the Data Fusion Committee of the IEEE GRSS.

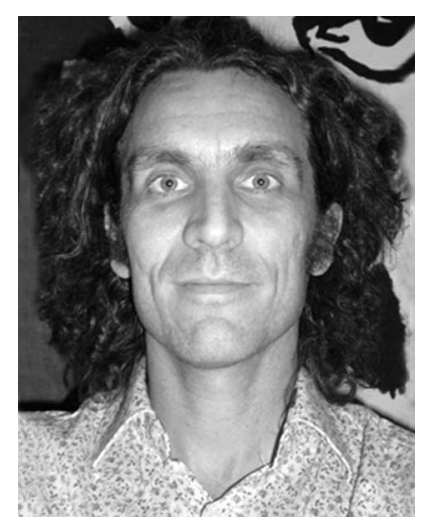

Jocelyn Chanussot (SM'04) received the M.Sc. degree in electrical engineering from the Grenoble Institute of Technology (INP Grenoble), Grenoble, France, in 1995, and the Ph.D. degree from Savoie University, Annecy, France, in 1998.

In 1999, he was with the Geography Imagery Perception Laboratory for the Delegation Generale de l'Armement (French National Defense Department). Since 1999, he has been with INP Grenoble as an Assistant Professor from 1999 to 2005, an Associate Professor from 2005 to 2007 , and currently a Professor of signal and image processing. He is conducting his research at the Grenoble Images Speech Signals and Automatics Laboratory, INP Grenoble. His research interests include image analysis, multicomponent image processing, nonlinear filtering, and data fusion in remote sensing.

Dr. Chanussot is an Associate Editor for the IEEE TRANSACTIONS ON GEOSCIENCE AND Remote Sensing and for Pattern Recognition. He is the Cochair of the GRS Data Fusion Technical Committee and a member of the Machine Learning for Signal Processing Technical Committee of the IEEE Signal Processing Society. He is the Founding President of the IEEE Geoscience and Remote Sensing French chapter. 